\title{
Eddy currents in MRI gradient coils configured as rings and patches
}

\author{
J. M. B. Kroot • S. J. L. van Eijndhoven • \\ A. A. F. van de Ven
}

Received: 2 June 2010 / Accepted: 13 February 2011 / Published online: 26 March 2011

(C) The Author(s) 2011. This article is published with open access at Springerlink.com

\begin{abstract}
The current distribution in a set of parallel rings and patches, called islands, positioned at the surface of a cylinder, is investigated. The current is driven by an externally applied source current. The islands are rectangular pieces of copper (patches) placed in parallel between the rings. The eddy currents in the islands induce currents in the rings that vary in the tangential direction. From the quasi-static Maxwell equations, an integral equation for the current distribution in the strips is derived. The Galerkin method, using global basis functions, is applied to solve this integral equation. It shows fast convergence. The global basis functions are Legendre polynomials in the axial direction and $2 \pi$-periodic trigonometric functions in the tangential direction. The Legendre polynomials efficiently cope with the singularity of the kernel function of the integral equation. Explicit numerical results are shown for three configurations. Apart from the current distributions, the resistance and self-inductance of the three systems of rings and islands are computed. The resulting tool can be used to qualitatively understand eddy currents in $z$-gradient coils, and as such enable the incorporation of eddy currents in the optimization of gradient-coil design.
\end{abstract}

Keywords Eddy currents · Gradient coils $\cdot$ Legendre polynomials $\cdot$ MRI-scanning $\cdot$ Patches

\section{Introduction}

Magnetic Resonance Imaging (MRI) is a scanning technique that plays a prominent role in medical diagnostics. It provides images of cross sections of a body, taken from any angle; see [1,2]. The selection of a slice is realized by the gradient coils. A gradient coil consists of copper strips wrapped around a cylinder. In practice, a coil is made by winding a metal plate around a cylinder, after which slits are cut to form strips. These strips are designed to have such a shape that they form a single coil. The strips carry an electric current that is driven by an externally applied source current. Due to mutual magnetic coupling, the current is not uniformly distributed and eddy currents that affect the quality of the image arise. The literature on eddy currents in gradient coils is limited. There are some notable books on electromagnetic fields that provide background on eddy currents. The book by Tegopoulos and Kriezis [3] is concerned with analytical methods applied mainly to two-dimensional configurations. Stoll [4] presents both analytical and numerical methods; especially the finite-difference method is widely documented.

J. M. B. Kroot $(\varangle)$ · S. J. L. van Eijndhoven · A. A. F. van de Ven Eindhoven University of Technology, P.O. Box 513, 5600 MB Eindhoven, The Netherlands e-mail: j.m.b.kroot@gmail.com 
The book by Lammeraner and Stafl [5] is devoted to an analytical approach, but covers only one-dimensional configurations. The design of gradient coils is an important link in the development of MRI-scanners. In [6,7], Roméo and Hoult describe methods to determine the positions of wire turns on a coil and the intensities of the currents to obtain an optimal gradient field. Turner [8] applies a target-field method in which the desired field is specified, and the corresponding current distribution on the cylindrical surface is computed. Optimization algorithms are used in the conjugate-gradient method by Wong and Jesmanowicz [9], in the simulated-annealing method by Crozier and Doddrell [10], and in the stream-functions method by Tomasi [11] in which the current distribution is discretized by use of one-dimensional wires. Trakic et al. [12] incorporate transient eddy currents in a numerical study of a coil-optimization process; they modify a gradient-coil design by combining the fields created by the coil and the eddy currents to generate a spatially homogeneous magnetic gradient field.

In a previous study, a framework of models has been established to describe the current distribution in gradient coils. In [13], as a starting model, the current distribution is determined in a parallel set of plane rectangular strips. This study describes the derivation of a Fredholm integral equation of the second kind with logarithmically singular kernel. Application of the Galerkin method with Legendre polynomials as basis functions leads to an efficient algorithm. In reality, gradient coils consist of copper strips wrapped around a cylinder. Therefore, in [14], the current distribution is determined in a parallel set of rings on a cylinder. The current then flows only in the tangential direction and distributes non-uniformly over the axial direction. The solution method is similar to the approach in [13], but allowing currents to flow around the cylinder. This model can be used to determine the current distribution as well as the corresponding magnetic field of the so-called $z$-gradient coil. A $z$-gradient coil creates a magnetic field with gradient in the axial $(z-)$ direction. Gradient coils that produce a magnetic gradient field in perpendicular $(x-$, or $y$-) direction are called $x$-gradient coils and $y$-gradient coils. They consist of strips that are placed spirally on the surface of a cylinder. For curved circular strips of width much smaller than the radius of the cylinder, one may locally replace the curved circular strip by a tangent-plane circular strip, as described in [15].

In an MRI-scanner, the $x$-, $y$-, and $z$-gradient coils are installed together. By powering the gradient coils in combination, it is possible to generate magnetic-field gradients in any direction. As such, MRI images can be produced in planes that can be arbitrarily oriented. Due to mutual induction, extra eddy currents may occur in the coils. These unwanted eddy currents disturb the magnetic field, which results in distorted MRI images. Disturbances are also caused by slits that are cut in the strips. These slits are necessary to direct the current, and to control the impedance and heat dissipation of the coils; see, e.g. [6]. Thus, it is important to be able to simulate the current distribution to design gradient coils in which unwanted eddy currents are prevented.

This article presents an extension of the ring model for the current distribution in the $z$-gradient coil. In the extended model, rectangular copper patches are placed between the rings. The patches are like islands in which eddy currents are induced by changing electromagnetic fields. Additionally, eddy currents in the rings are induced, because they experience the presence of the islands. The currents do not flow only in the tangential direction, as they do in a set of parallel rings, see [14], but also in the axial direction. This is a result of the fact that the current density is divergence free, as follows from the continuity equation. The objective of this article is to present a mathematical model for currents flowing on a cylindrical surface in both the tangential and the axial direction. As an analytical model, it can be considered as complementary to the existing literature in which mainly numerical models and coil-development (optimization) models are presented. The rings and patches are not used to mimic an actual $z$-gradient coil. However, the geometry of the model is general: any set of rings and patches can be used as input in the model. The returned output gives insight in the qualitative behavior of the currents in the coil and the corresponding magnetic field and power dissipation.

In Section 2, we briefly describe the set-up of the model. The geometry of the strips on the cylinder is defined together with the governing Maxwell equations in dimensionless form. Section 3 describes the transformation of the Maxwell equations into an integral equation. Different representations of the singular kernel function are given, leading to the Fourier-cosine series representation of the kernel function that is needed for the numerical calculations. The solution procedure in Section 4 explains how the Galerkin method is applied. Global basis functions, defined in Section 4.1, efficiently cope with the logarithmically singular kernel function. The Galerkin method yields a matrix equation; the determination of each element of the matrix kernel is described in Section 4.2. Section 5 presents 
numerical results for several sets of rings and islands. The geometries used in the numerical examples are chosen such that they can be compared with the ring examples presented in [14]. The examples are not meant to mimic realistic $z$-gradient coils. Section 6 summarizes the conclusions.

\section{Model formulation}

Consider a set of $N_{r}$ parallel coaxial circular conducting strips, or rings, and $N_{i}$ rectangular patches or islands. These conductors lie on the same virtual cylinder $S_{c}$, defined in terms of cylindrical coordinates as

$S_{c}=\{(r, \varphi, z) \mid r=R,-\pi \leq \varphi \leq \pi,-\infty<z<\infty\}$.

Each separate ring or island is of uniform width (in the $z$-direction), with thickness $h$ (in the $r$-direction) being the same for every conductor. One central line of each island is parallel to the rings. Since the strips are very thin, the current distribution in the $r$-direction can be considered uniform; see [13, Sect. 2]. This means that the current can be modeled as flowing on the surface of the cylinder, i.e., on $r=R$, as if the strips are of infinitesimal thickness. The strips occupy the surface $S_{\cup}=S_{r}+S_{i}$ on the cylinder, with $S_{r}$ being the total surface of the rings and $S_{i}$ the total surface of the islands. We indicate the surface of one ring or one island by $S_{q}$, where the strips are numbered successively by $q=1,2, \ldots, N_{r}$, for the rings, and $q=N_{r}+1, N_{r}+2, \ldots, N_{r}+N_{i}$, for the islands. Hence,

$S_{r}=\sum_{q=1}^{N_{r}} S_{q}, \quad S_{i}=\sum_{q=1}^{N_{i}} S_{N_{r}+q}$,

where

$S_{q}=\left\{(r, \varphi, z) \mid r=R,-\pi \leq \varphi \leq \pi, z_{0}^{(q)} \leq z \leq z_{1}^{(q)}\right\}$,

for $q=1,2, \ldots, N_{r}$, and

$S_{q}=\left\{(r, \varphi, z) \mid r=R, \varphi_{0}^{(q)} \leq \varphi \leq \varphi_{1}^{(q)}, z_{0}^{(q)} \leq z \leq z_{1}^{(q)}\right\}$,

for $q=N_{r}+1, N_{r}+2, \ldots, N_{r}+N_{i}$. More islands can be present between two rings. A configuration of three rings and four islands is depicted in Fig. 1. In this example, the islands have the same axial beginning and ending positions $z_{0}^{(q)}$ and $z_{1}^{(q)}, q=4,5,6,7$.

The source currents are exclusively applied to the rings, but, although not applied to the islands, these source currents will excite the whole system. Each source current is composed of pulses that can be represented by a limited series of time-harmonics with relatively low frequencies, $f<10^{4} \mathrm{~Hz}$. This allows for a quasi-static approach. We assume that the source currents change harmonically in time with a frequency $\omega=2 \pi f$; the total response is determined by superposition.

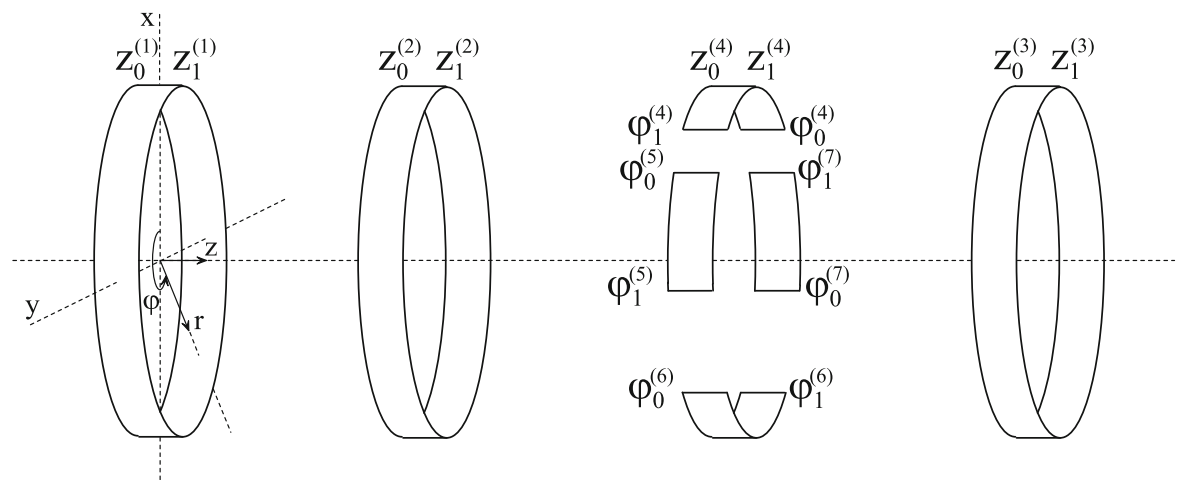

Fig. 1 The geometry of a set of three rings and four islands; here, the islands have the same axial begin and end positions 
The current on a ring flows completely around the cylinder, whereas on an island it cannot. Since the current distribution has to be divergence free, the only possible way for a current to flow on an island is to form closed cycles within the island. In the results of Sect. 5, the occurrence of such eddies is illustrated.

To obtain a mathematical description of the problem, we start from Maxwell's theory as described by Stratton [16]. A dimensionless form of the Maxwell equations is obtained by scaling the radial and axial distances by the radius of the cylinder $R$, the magnetic field, and the current density by the average current through all rings $j^{c}$ (for its definition see (2.15), further on), and the vector potential by a factor $\mu_{0} R j^{c}$, where $\mu_{0}=4 \pi \cdot 10^{-7} \mathrm{H} / \mathrm{m}$ is the magnetic permeability in free space. This yields the first-order differential equations:

$\nabla \times \mathbf{A}=\mathbf{H}, \quad \nabla \times \mathbf{H}=\mathbf{0}, \quad \nabla \cdot \mathbf{A}=0, \quad \nabla \cdot \mathbf{H}=0$,

while use of the gauge $\nabla \cdot \mathbf{A}=0$, gives

$\Delta \mathbf{A}=\mathbf{0}$.

where, $\mathbf{H}$ and $\mathbf{A}$ are the dimensionless magnetic field and vector potential, respectively. These equations hold in both the inner region and the outer region of the cylinder. Across the cylinder, the following jump conditions are prescribed:

$H_{r}^{-}(1, \varphi, z)=H_{r}^{+}(1, \varphi, z)$,

$H_{\varphi}^{+}(1, \varphi, z)-H_{\varphi}^{-}(1, \varphi, z)=j_{z}(\varphi, z)$,

$H_{z}^{+}(1, \varphi, z)-H_{z}^{-}(1, \varphi, z)=-j_{\varphi}(\varphi, z)$,

$\mathbf{A}^{-}(1, \varphi, z)=\mathbf{A}^{+}(1, \varphi, z)$.

The superscripts $\mp$ represent the fields just inside or outside the cylinder $r=1$, respectively.

The conditions at infinity require

$\mathbf{H} \rightarrow \mathbf{0}, \quad \nabla \times \mathbf{A} \rightarrow \mathbf{0}, \quad$ for $|\mathbf{x}| \rightarrow \infty$.

Although the total current density $\mathbf{j}$ has components in both the $\varphi$ - and $z$-directions, they are induced by a prescribed source current in the rings that has only a $\varphi$-component. In the next section, we derive integral equations for $j_{\varphi}$ and $j_{z}$. We solve $j_{\varphi}=j_{\varphi}(\varphi, z)$ and derive the $z$-component from the divergence relation for the current density, i.e.,

$\nabla \cdot \mathbf{j}=0, \quad \Longrightarrow \frac{\partial j_{z}}{\partial z}=-\frac{\partial j_{\varphi}}{\partial \varphi}$.

Since the current cannot flow across the edges of the strips, the normal components of the current densities at the edges must be zero. For the rings, this implies for $q \in\left\{1, \ldots, N_{r}\right\}$

$j_{z}\left(\varphi, z_{0}^{(q)}\right)=j_{z}\left(\varphi, z_{1}^{(q)}\right)=0$,

whereas for the islands, $q \in\left\{N_{r}+1, \ldots, N_{r}+N_{i}\right\}$, we have, besides (2.13), the additional condition

$j_{\varphi}\left(\varphi_{0}^{(q)}, z\right)=j_{\varphi}\left(\varphi_{1}^{(q)}, z\right)=0$.

As in the model presented in [14], the set of rings is subdivided into $L$ groups, each of which has a prescribed total current $I_{l}, l=1, \ldots, L$ and is driven by a separate source. The sum of the widths of all rings in group $l$ is denoted by $D_{l}$. The cross-sectional averaged current density through all rings $j^{c}$ (needed later on in Eq. 4.9) is then defined by

$j^{c}=\frac{\sum_{l=1}^{L} I_{l}}{\sum_{l=1}^{L} D_{l}}$.

The source current is incorporated in the model via Ohm's law (including source currents; see [15, Eq. 6])

$i \kappa A_{\varphi}^{-}(1, \varphi, z)=\mathrm{i} \kappa A_{\varphi}^{+}(1, \varphi, z)=j_{\varphi}(\varphi, z)-j_{\varphi}^{s}(\varphi, z)$,

$i \kappa A_{z}^{-}(1, \varphi, z)=\mathrm{i} \kappa A_{z}^{+}(1, \varphi, z)=j_{z}(\varphi, z)$, 
where $\kappa=h \sigma \mu_{0} \omega R$ is a system parameter, and $j_{\varphi}^{s}$ denotes the source current density applied to the rings. The electric conductivity of copper is $\sigma=5.88 \cdot 10^{7} \Omega^{-1} \mathrm{~m}^{-1}$.

The prescribed source current density is $2 \pi$-periodic (i.e., $\left.j_{\varphi}^{s}(\varphi, z)=\sum_{n=-\infty}^{\infty} j_{\varphi}^{(n), s}(z) \mathrm{e}^{\mathrm{i} i \varphi}\right)$, and consequently, all the induced vector fields, like $\mathbf{A}$ and $\mathbf{j}$ as well.

\section{Integral formulation}

In this section, we derive from the set of dimensionless differential equations of Sect. 2 an integral equation for the current-density distribution $\mathbf{j}=j_{\varphi}(\varphi, z) \mathbf{e}_{\varphi}+j_{z}(\varphi, z) \mathbf{e}_{z}$. Subsequently, the resulting kernel function is transformed into a form that is useful for handling the singularities in the numerical calculations.

\subsection{Derivation of the integral equation}

For the derivation of the integral equation for the current-density distribution, we introduce the complex Fourierseries expansions of the $2 \pi$-periodic vector potential $\mathbf{A}$, the magnetic field $\mathbf{H}$, and the current density $\mathbf{j}$, according to

$$
\begin{aligned}
& A_{q}(r, \varphi, z)=\sum_{n=-\infty}^{\infty} A_{q}^{(n)}(r, z) \mathrm{e}^{\mathrm{i} n \varphi}, \quad(\text { for } q=r, \varphi, z), \\
& H_{q}(r, \varphi, z)=\sum_{n=-\infty}^{\infty} H_{q}^{(n)}(r, z) \mathrm{e}^{\mathrm{i} n \varphi}, \quad(\text { for } q=r, \varphi, z), \\
& j_{q}(\varphi, z)=\sum_{n=-\infty}^{\infty} j_{q}^{(n)}(z) \mathrm{e}^{\mathrm{i} n \varphi}, \quad(\text { for } q=\varphi, z) .
\end{aligned}
$$

To the equations obtained by inserting (3.1) into (2.6), we apply the Fourier integral transformation (see e.g., [17, Sect.2.3]) with respect to the variable $z$. A function $g(r, z)$ is Fourier transformed to $\hat{g}(r, p)$, according to

$\hat{g}(r, p)=\mathcal{F}_{z}\{g(r, \cdot)\}(p)=\int_{-\infty}^{\infty} g(r, z) \mathrm{e}^{-\mathrm{i} p z} \mathrm{~d} z$.

Introducing the Fourier integral transforms $\hat{A}_{q}^{(n)}(r, p)=\mathcal{F}_{z}\left\{A_{q}^{(n)}(r, \cdot)\right\}(p)$, for both the inner and outer regions, we obtain from (2.6),

$$
\begin{aligned}
& -\left(\frac{n^{2}}{r^{2}}+p^{2}\right) \hat{A}_{z}^{(n)}+\frac{1}{r} \frac{\partial}{\partial r}\left(r \frac{\partial \hat{A}_{z}^{(n)}}{\partial r}\right)=0, \\
& -\left(\frac{n^{2}+1}{r^{2}}+p^{2}\right) \hat{A}_{\varphi}^{(n)}+\frac{1}{r} \frac{\partial}{\partial r}\left(r \frac{\partial \hat{A}_{\varphi}^{(n)}}{\partial r}\right)+\frac{2 n \mathrm{i}}{r^{2}} \hat{A}_{r}^{(n)}=0, \\
& -\left(\frac{n^{2}+1}{r^{2}}+p^{2}\right) \hat{A}_{r}^{(n)}+\frac{1}{r} \frac{\partial}{\partial r}\left(r \frac{\partial \hat{A}_{r}^{(n)}}{\partial r}\right)-\frac{2 n \mathrm{i}}{r^{2}} \hat{A}_{\varphi}^{(n)}=0 .
\end{aligned}
$$

In the derivation of (3.5)-(3.7), the radiation conditions at infinity are used; see (2.11). The general solution can be expressed in terms of $I_{n}$ and $K_{n}$, which are modified Bessel functions of order $n$ of the first and second kind, respectively (see, e.g. [18,19]). This yields

$$
\begin{aligned}
& \hat{A}_{r}^{(n)-}(r, p)=-\mathrm{i} C_{1}^{(n)}(p) I_{n-1}(|p| r)+\mathrm{i} C_{2}^{(n)}(p) I_{n+1}(|p| r), \\
& \hat{A}_{\varphi}^{(n)-}(r, p)=C_{1}^{(n)}(p) I_{n-1}(|p| r)+C_{2}^{(n)}(p) I_{n+1}(|p| r), \\
& \hat{A}_{z}^{(n)-}(r, p)=C_{3}^{(n)}(p) I_{n}(|p| r),
\end{aligned}
$$


for the inner region (denoted by the superscript ${ }^{-}$), with arbitrary functions $C_{1}^{(n)}(p), C_{2}^{(n)}(p)$ and $C_{3}^{(n)}(p)$. For the outer region (denoted by the superscript ${ }^{+}$), we obtain

$$
\begin{aligned}
& \hat{A}_{r}^{(n)+}(r, p)=-\mathrm{i} C_{4}^{(n)}(p) K_{n-1}(|p| r)+\mathrm{i} C_{5}^{(n)}(p) K_{n+1}(|p| r), \\
& \hat{A}_{\varphi}^{(n)+}(r, p)=C_{4}^{(n)}(p) K_{n-1}(|p| r)+C_{5}^{(n)}(p) K_{n+1}(|p| r), \\
& \hat{A}_{z}^{(n)+}(r, p)=C_{6}^{(n)}(p) K_{n}(|p| r),
\end{aligned}
$$

with arbitrary functions $C_{4}^{(n)}(p), C_{5}^{(n)}(p)$ and $C_{6}^{(n)}(p)$. Using $\mathbf{H}=\nabla \times \mathbf{A}$, the boundary conditions (2.7)-(2.10), and the divergence-free condition (2.12), we obtain

$$
\begin{aligned}
& C_{1}^{(n)}(p)=\frac{1}{2} K_{n-1}(|p|) \hat{\mathrm{j}}_{\varphi}^{(n)}(p), \quad C_{4}^{(n)}(p)=\frac{1}{2} I_{n-1}(|p|) \hat{\mathrm{j}}_{\varphi}^{(n)}(p), \\
& C_{2}^{(n)}(p)=\frac{1}{2} K_{n+1}(|p|) \hat{\mathrm{j}}_{\varphi}^{(n)}(p), \quad C_{5}^{(n)}(p)=\frac{1}{2} I_{n+1}(|p|) \hat{\mathrm{j}}_{\varphi}^{(n)}(p), \\
& C_{3}^{(n)}(p)=\frac{1}{2} K_{n}(|p|) \hat{\mathrm{j}}_{z}^{(n)}(p), \quad C_{6}^{(n)}(p)=\frac{1}{2} I_{n}(|p|) \hat{\mathrm{j}}_{z}^{(n)}(p) .
\end{aligned}
$$

For the inverse Fourier transformation, we use the convolution principle. Both the limits, $r \uparrow 1$ for the inner region and $r \downarrow 1$ in the outer region, give the same results. We obtain

$$
\begin{aligned}
& A_{r}(1, \varphi, z)=\iint_{S_{c}} \mathcal{K}_{r}(\varphi-\theta, z-\zeta) j_{\varphi}(\theta, \zeta) \mathrm{d} \theta \mathrm{d} \zeta, \\
& A_{\varphi}(1, \varphi, z)=\iint_{S_{c}} \mathcal{K}_{\varphi}(\varphi-\theta, z-\zeta) j_{\varphi}(\theta, \zeta) \mathrm{d} \theta \mathrm{d} \zeta, \\
& A_{z}(1, \varphi, z)=\iint_{S_{c}} \mathcal{K}_{z}(\varphi-\theta, z-\zeta) j_{z}(\theta, \zeta) \mathrm{d} \theta \mathrm{d} \zeta,
\end{aligned}
$$

valid on the whole cylinder $S_{c}$, with kernel functions

$$
\begin{aligned}
& \mathcal{K}_{r}(\varphi, z)=\frac{1}{8 \pi^{2}} \sum_{n=-\infty}^{\infty} \int_{-\infty}^{\infty}\left[I_{n-1}(|p|) K_{n-1}(|p|)-I_{n+1}(|p|) K_{n+1}(|p|)\right] \mathrm{e}^{\mathrm{i} p z} \mathrm{e}^{\mathrm{i} n \varphi} \mathrm{d} p, \\
& \mathcal{K}_{\varphi}(\varphi, z)=\frac{1}{8 \pi^{2}} \sum_{n=-\infty}^{\infty} \int_{-\infty}^{\infty}\left[I_{n-1}(|p|) K_{n-1}(|p|)+I_{n+1}(|p|) K_{n+1}(|p|)\right] \mathrm{e}^{\mathrm{i} p z} \mathrm{e}^{\mathrm{i} n \varphi} \mathrm{d} p
\end{aligned}
$$

and

$$
\mathcal{K}_{z}(\varphi, z)=\frac{1}{4 \pi^{2}} \sum_{n=-\infty}^{\infty} \int_{-\infty}^{\infty} I_{n}(|p|) K_{n}(|p|) \mathrm{e}^{\mathrm{i} p z} \mathrm{e}^{\mathrm{i} n \varphi} \mathrm{d} p .
$$

Finally, the integral equations for the current density $\mathbf{j}=j_{\varphi}(\varphi, z) \mathbf{e}_{\varphi}+j_{z}(\varphi, z) \mathbf{e}_{z}$ are obtained from (2.16) and (2.17), in which the currents are restricted to the surface $S \cup$, yielding

$$
\begin{aligned}
& \mathrm{i} \kappa \iint_{S \cup} \mathcal{K}_{\varphi}(\varphi-\theta, z-\zeta) j_{\varphi}(\theta, \zeta) \mathrm{d} \theta \mathrm{d} \zeta=j_{\varphi}(\varphi, z)-j_{\varphi}^{s}(\varphi, z), \\
& \mathrm{i} \kappa \iint_{S \cup} \mathcal{K}_{z}(\varphi-\theta, z-\zeta) j_{z}(\theta, \zeta) \mathrm{d} \theta \mathrm{d} \zeta=j_{z}(\varphi, z) .
\end{aligned}
$$

Note that the currents are restricted to $S \cup$ by the electric conductivity, $\sigma$, which is set equal to zero on the cylindrical surface between the strips. The linear integral equations (3.23) and (3.24) are integral equations of the second 
kind. The important advantage of these integral equations is that they are valid for an arbitrary set of conductors $S \cup$ on the surface of the cylinder. In this article, we focus on solving the equations for rings and patches. We show the derivation of the solution for $j_{\varphi}$, since the main current is in the $\varphi$-direction and $j_{z}$ automatically follows if the basis functions are chosen in such a way that (2.12)-(2.14) are satisfied. This will be demonstrated in Sect. 4.

\subsection{Transformation of the kernel function}

Next, we transform the kernel function of integral equation (3.23) into a form that is useful for handling the singularities in the numerical calculations. The transformation first uses the following formula from [18, p. 315]:

$$
\frac{\pi}{2} \int_{0}^{\infty} J_{\mu}(a t) J_{v}(b t) \mathrm{e}^{-|c| t} t^{\lambda} \mathrm{d} t=\int_{0}^{\infty} K_{\mu}(a t) I_{\nu}(b t) \cos \left(|c| t+\frac{1}{2}(\mu-v+\lambda) \pi\right) t^{\lambda} \mathrm{d} t,
$$

where $J_{k}$ is the $k$ th-order Bessel function of the first kind. With (3.25), we derive

$$
\begin{aligned}
\int_{-\infty}^{\infty} & {\left[I_{k-1}(|p|) K_{k-1}(|p|)+I_{k+1}(|p|) K_{k+1}(|p|)\right] \mathrm{e}^{\mathrm{i} p z} \mathrm{~d} p } \\
= & 2 \int_{0}^{\infty}\left[I_{k-1}(s) K_{k-1}(s)+I_{k+1}(s) K_{k+1}(s)\right] \cos (s z) \mathrm{d} s \\
= & \pi \int_{0}^{\infty}\left[J_{k-1}^{2}(s)+J_{k+1}^{2}(s)\right] \mathrm{e}^{-s|z|} \mathrm{d} s,
\end{aligned}
$$

such that

$$
\begin{aligned}
\mathcal{K}_{\varphi}(\varphi, z) & =\frac{1}{8 \pi} \sum_{k=-\infty}^{\infty} \int_{0}^{\infty}\left[J_{k-1}^{2}(s)+J_{k+1}^{2}(s)\right] \mathrm{e}^{-s|z|} \mathrm{e}^{\mathrm{i} k \varphi} \mathrm{d} s \\
& =\frac{1}{4 \pi} \sum_{k=-\infty}^{\infty} \int_{0}^{\infty} J_{k}^{2}(s) \cos (\varphi) \mathrm{e}^{-s|z|} \mathrm{e}^{\mathrm{i} k \varphi} \mathrm{d} s .
\end{aligned}
$$

Next, formula (13.22.2) from [19] tells us that

$$
\int_{0}^{\infty} \mathrm{e}^{-s|z|} J_{p}^{2}(s) \mathrm{d} s=\frac{1}{\pi} Q_{p-\frac{1}{2}}\left(\frac{2+z^{2}}{2}\right) .
$$

The functions $Q_{p-\frac{1}{2}}, p \geq 0$, are Legendre functions of the second kind of odd-half-integer order. Substitution of (3.28) in (3.27) yields

$\mathcal{K}_{\varphi}(\varphi, z)=\frac{\cos (\varphi)}{4 \pi^{2}}\left(Q_{-\frac{1}{2}}(\chi)+2 \sum_{p=1}^{\infty} \cos (p \varphi) Q_{p-\frac{1}{2}}(\chi)\right)$,

where we used that $Q_{-p-\frac{1}{2}}(\chi)=Q_{p-\frac{1}{2}}(\chi)$ and $\chi=\left(2+z^{2}\right) / 2$. Reordering the summation steps in (3.29), we arrive at the Fourier-cosine series representation of $\mathcal{K}_{\varphi}(\varphi, z)$

$\mathcal{K}_{\varphi}(\varphi, z)=\frac{1}{4 \pi^{2}}\left(Q_{\frac{1}{2}}(\chi)+\sum_{p=1}^{\infty} \cos (p \varphi)\left(Q_{p-\frac{3}{2}}(\chi)+Q_{p+\frac{1}{2}}(\chi)\right)\right)$,

as we will use in the solution procedure in the next section. 
For all $p \geq 0$, the functions $Q_{p-\frac{1}{2}}(\chi)$ are logarithmically singular at $\chi=1$ (i.e., $z=0$ ), as can be seen from the asymptotic expansion of $Q_{p-\frac{1}{2}}(\chi)$ for $z \rightarrow 0$; see, e.g., [20, Eq. 4.8.2], [21, Eq. 59.9.2]:

$Q_{p-\frac{1}{2}}(\chi)=-\log |z|-\gamma+\log 2-\Psi^{(0)}\left(\frac{2 p+1}{2}\right)+\mathcal{O}\left(z^{2}\right)$.

where, $\gamma$ is Euler's constant, and $\Psi^{(0)}$ is the polygamma function; see [22]. Note that for $p=1$ the expansion is the same as the one obtained in the model of rings only; see [14]. Moreover, each cosine mode in (3.30) has a logarithmic singularity at $z=0$, which is of the same magnitude for all $p \geq 0$.

We conclude by showing another transformation of the kernel function that yields a more familiar expression. We start from (3.27). If we use the following equations from [19, pp. 358, 384],

$$
\begin{aligned}
\sum_{k=-\infty}^{\infty} J_{k}^{2}(s) \mathrm{e}^{\mathrm{i} k \varphi} & =J_{0}\left(2 s \sin \frac{\varphi}{2}\right), \\
\int_{0}^{\infty} J_{0}(b t) \mathrm{e}^{-|a| t} \mathrm{~d} t & =\frac{1}{\sqrt{a^{2}+b^{2}}},
\end{aligned}
$$

then (3.27) results in

$$
\begin{aligned}
\mathcal{K}_{\varphi}(\varphi, z) & =\frac{1}{4 \pi} \sum_{k=-\infty}^{\infty} \int_{0}^{\infty} J_{k}^{2}(s) \cos (\varphi) \mathrm{e}^{-s|z|} \mathrm{e}^{\mathrm{i} k \varphi} \mathrm{d} s \\
& =\frac{\cos (\varphi)}{4 \pi} \int_{0}^{\infty} J_{0}\left(2 s \sin \frac{\varphi}{2}\right) \mathrm{e}^{-s|z|} \mathrm{d} s \\
& =\frac{1}{4 \pi} \frac{\cos (\varphi)}{\sqrt{z^{2}+4 \sin ^{2}(\varphi / 2)}},
\end{aligned}
$$

which is the $\varphi$-component of Biot-Savart's kernel function for currents flowing on the surface of a cylinder. The $z$-component can be derived analogously. Note that (3.31) will be used further in this article to deal with the logarithmic singularity of the kernel function explicitly.

\section{Solution procedure}

In this section, we explain how we solve $j_{\varphi}(\varphi, z)$ from (3.23), using the representation (3.30) for the kernel. We apply the Galerkin method with basis functions that take into account (2.13) and (2.14), and accurately deal with the singular behavior of the kernel function at $(\varphi, z)=(0,0)$.

\subsection{Basis functions}

We want to use global basis functions, i.e., functions defined globally on the rings and islands. Due to the logarithmic singularity of the kernel function in the $z$-coordinate we choose Legendre polynomials of the first kind for this direction. These polynomials enable us to use analytical solutions for the singular integrals (this will be demonstrated in (4.25)). In the $\varphi$-direction, we must use $2 \pi$-periodic trigonometric functions.

The $\varphi$-component of the current-density distribution on all strips is decomposed into $j_{r, \varphi}$ for the rings and $j_{i, \varphi}$ for the islands, and represented by a two-vector, such that we can write (3.23) as an operator matrix equation, according to

$$
\left(\begin{array}{c}
j_{r, \varphi} \\
j_{i, \varphi}
\end{array}\right)-\mathrm{i} \kappa\left(\begin{array}{ll}
\mathrm{K}_{r r} & \mathrm{~K}_{r i} \\
\mathrm{~K}_{i r} & \mathrm{~K}_{i i}
\end{array}\right)\left(\begin{array}{l}
j_{r, \varphi} \\
j_{i, \varphi}
\end{array}\right)=\left(\begin{array}{c}
j_{r, \varphi}^{s} \\
0
\end{array}\right) .
$$


The operator $\mathrm{K}_{r r}$ represents the inductive effects between the rings mutually, while $\mathrm{K}_{i i}$ represents those effects between the islands mutually, and $\mathrm{K}_{r i}$ and $\mathrm{K}_{i r}$ those between rings and islands. System parameter $\kappa$ is related to the frequency and defined below (2.17) as $\kappa=h \sigma \mu_{0} \omega R$.

Taking into account the boundary conditions $(\mathbf{j} \cdot \mathbf{n})=0$ and the divergence-free current relation $\nabla \cdot \mathbf{j}=0$, we arrive at the following expansion for the (dimensionless) current density:

1. On the rings, numbered $q$, with $q \in\left\{1, \ldots, N_{r}\right\}$, and $z \in\left[z_{0}^{(q)}, z_{1}^{(q)}\right]$ :

$$
\begin{aligned}
j_{r, \varphi}^{(q)}(\varphi, z)= & \left\{\sum_{m=1}^{\infty} \sum_{n=1}^{\infty}\left(\alpha_{m n}^{(q)} \cos m \varphi+\beta_{m n}^{(q)} \sin m \varphi\right) P_{n}\left(\frac{z-c_{z}^{(q)}}{d_{z}^{(q)}}\right)\right. \\
& \left.+\sum_{n=1}^{\infty} \alpha_{0 n}^{(q)} P_{n}\left(\frac{z-c_{z}^{(q)}}{d_{z}^{(q)}}\right)+\alpha_{00}^{(q)}\right\} \mathbf{1}_{\left[z_{0}^{(q)}, z_{1}^{(q)}\right]}, \\
j_{r, z}^{(q)}(\varphi, z)= & \left\{\sum_{m=1}^{\infty} \sum_{n=1}^{\infty} m d_{z}^{(q)}\left(-\beta_{m n}^{(q)} \cos m \varphi+\alpha_{m n}^{(q)} \sin m \varphi\right) Z_{n}\left(\frac{z-c_{z}^{(q)}}{d_{z}^{(q)}}\right)\right\} \mathbf{1}_{\left[z_{0}^{(q)}, z_{1}^{(q)}\right]},
\end{aligned}
$$

where

$$
\begin{aligned}
& c_{z}^{(q)}=\frac{z_{1}^{(q)}+z_{0}^{(q)}}{2}, \quad d_{z}^{(q)}=\frac{z_{1}^{(q)}-z_{0}^{(q)}}{2}, \\
& Z_{n}(z)=\int_{-1}^{z} P_{n}(\zeta) \mathrm{d} \zeta=\frac{1}{2 n+1}\left[P_{n+1}(z)-P_{n-1}(z)\right] .
\end{aligned}
$$

where $\mathbf{1}_{\left[z_{0}^{(q)}, z_{1}^{(q)}\right]}$ is the characteristic function, i.e., the function equal to one on the interval $\left[z_{0}^{(q)}, z_{1}^{(q)}\right]$, and zero otherwise. The terms in the second line of (4.2) correspond to the basis functions used for the rings-only case (see [14]) and correspond to divergence-free currents without a $j_{z}$-component. The terms with $m>0$ account for currents with a $j_{z}$-component. They occur due to mutual induction from the islands.

2. On the islands, numbered $q$, with $q \in\left\{N_{r}+1, \ldots, N_{r}+N_{i}\right\}$, and $\varphi \in\left[\varphi_{0}^{(q)}, \varphi_{1}^{(q)}\right], z \in\left[z_{0}^{(q)}, z_{1}^{(q)}\right]$ :

$$
\begin{aligned}
& j_{i, \varphi}^{(q)}(\varphi, z)=\sum_{m=1}^{\infty} \sum_{n=1}^{\infty} \alpha_{m n}^{(q)} \sin \left(\frac{m \pi\left(\varphi-\varphi_{0}^{(q)}\right)}{2 d_{\varphi}^{(q)}}\right) P_{n}\left(\frac{z-c_{z}^{(q)}}{d_{z}^{(q)}}\right) \mathbf{1}_{\left[z_{0}^{(q)}, z_{1}^{(q)}\right]} \mathbf{1}_{\left[\varphi_{0}^{(q)}, \varphi_{1}^{(q)}\right]}, \\
& j_{i, z}^{(q)}(\varphi, z)=-\sum_{m=1}^{\infty} \sum_{n=1}^{\infty} \frac{m \pi d_{z}^{(q)}}{2 d_{\varphi}^{(q)}} \alpha_{m n}^{(q)} \cos \left(\frac{m \pi\left(\varphi-\varphi_{0}^{(q)}\right)}{2 d_{\varphi}^{(q)}}\right) Z_{n}\left(\frac{z-c_{z}^{(q)}}{d_{z}^{(q)}}\right) \mathbf{1}_{\left[z_{0}^{(q)}, z_{1}^{(q)}\right]} \mathbf{1}_{\left[\varphi_{0}^{(q)}, \varphi_{1}^{(q)}\right]}
\end{aligned}
$$

where

$$
d_{\varphi}^{(q)}=\frac{\varphi_{1}^{(q)}-\varphi_{0}^{(q)}}{2}
$$

where $\mathbf{1}_{\left[\varphi_{0}^{(q)}, \varphi_{1}^{(q)}\right]}$ is the characteristic function on the interval $\left[\varphi_{0}^{(q)}, \varphi_{1}^{(q)}\right]$. Note that the representations (4.2), (4.3) and (4.6), (4.7) automatically satisfy the boundary conditions for zero normal current at the edges of the islands. Moreover, because $Z_{n}^{\prime}=P_{n}$, the divergence-free restriction is also automatically satisfied by these representations.

Because the source current acts on the $L$ groups of rings only, we introduce basis functions $\psi_{l}(z)$ that are equal to one on the rings of group $l, l=1, \ldots, L$, and zero otherwise. Projection of the $\varphi$-component of the current density in the rings on these basis functions yields (see [23, Eq. 4.140])

$\Pi j_{r, \varphi}=\sum_{l=1}^{L} \frac{\left(j_{r, \varphi}, \psi_{l}\right)}{\left(\psi_{l}, \psi_{l}\right)} \psi_{l}=\sum_{l=1}^{L} \frac{R \hat{I}_{l}}{D_{l}} \psi_{l}$, 
where $\Pi$ is the projection operator, (., .) denotes the inner product defined on $S_{c}$, and $\hat{I}_{l}=I_{l} /\left(j^{c} R\right)$. Consequently, $j_{r, \varphi}$ is decomposed into two parts according to

$j_{r, \varphi}=\Pi j_{r, \varphi}+(\mathrm{I}-\Pi) j_{r, \varphi}=\sum_{l=1}^{L} \frac{R \hat{I}_{l}}{D_{l}} \psi_{l}+j_{\perp}$.

where $j_{\perp}$ is in the orthogonal complement of the range of $\Pi$, i.e., $\left(j_{\perp}, \psi_{l}\right)=0$, for $l=1, \ldots, L$ (see e.g., [24, Sect. 3.3-3]). Multiplying the first row in (4.1) by $(\mathrm{I}-\Pi)$, we obtain

$$
\left(\begin{array}{c}
j_{\perp} \\
j_{i, \varphi}
\end{array}\right)-\mathrm{i} \kappa\left(\begin{array}{cc}
(\mathrm{I}-\Pi) \mathrm{K}_{r r} & (\mathrm{I}-\Pi) \mathrm{K}_{r i} \\
\mathrm{~K}_{i r} & \mathrm{~K}_{i i}
\end{array}\right)\left(\begin{array}{c}
j_{r, \varphi} \\
j_{i, \varphi}
\end{array}\right)=\left(\begin{array}{c}
0 \\
0
\end{array}\right),
$$

or equivalently,

$$
\left(\begin{array}{c}
j_{\perp} \\
j_{i, \varphi}
\end{array}\right)-\mathrm{i} \kappa\left(\begin{array}{cc}
(\mathrm{I}-\Pi) \mathrm{K}_{r r} & (\mathrm{I}-\Pi) \mathrm{K}_{r i} \\
\mathrm{~K}_{i r} & \mathrm{~K}_{i i}
\end{array}\right)\left(\begin{array}{c}
j_{\perp} \\
j_{i, \varphi}
\end{array}\right)=\mathrm{i} \kappa\left(\begin{array}{c}
\sum_{l=1}^{L} \frac{R \hat{I}_{l}}{D_{l}}(\mathrm{I}-\Pi) \mathrm{K} \psi_{l} \\
0
\end{array}\right) .
$$

The coefficient $\alpha_{00}^{(q)}$ in (4.2) represents the total current through the $q$ th ring, because all other basis functions integrate to zero. Within one group of rings, the coefficients $\alpha_{00}^{(q)}$ can still be different for each ring. The currents in the rings and islands can be approximated by (4.2), (4.3) and (4.6), (4.7), by taking a limited number of basis functions. We denote the highest degree of the basis functions on the $q$ th ring or island in the variable $\varphi$ or $z$ by $M_{\varphi}^{(q)}$ and $M_{z}^{(q)}$, respectively. To distinguish between the rings in one group, we need an additional number of $N_{r}-L$ basis functions for $j_{\perp}$. These functions are constructed by a linear combination of characteristic functions that are either 1 or 0 on a ring, in such a way that these basis functions are mutually orthogonal and orthogonal to all other basis functions. The number of basis functions for $j_{\perp}$ on the rings, denoted by $M_{r}$, is thus

$M_{r}=\sum_{q=1}^{N_{r}} M_{z}^{(q)}\left(2 M_{\varphi}^{(q)}+1\right)+N_{r}-L$,

whereas the number of basis functions on the islands, denoted by $M_{i}$, is

$M_{i}=\sum_{q=1}^{N_{i}} M_{z}^{\left(N_{r}+q\right)} M_{\varphi}^{\left(N_{r}+q\right)}$.

The total number of basis functions is equal to $M=M_{r}+M_{i}$. We denote each basis function by $\phi_{\mu}(\varphi, z)$, $\mu=1, \ldots, M$, and the coefficients by $\alpha_{r, \mu}$ for the rings and $\alpha_{i, \mu}$ for the islands, such that

$j_{\perp}(\varphi, z) \doteq \sum_{\mu=1}^{M_{r}} \alpha_{r, \mu} \phi_{\mu}(\varphi, z), \quad j_{i, \varphi}(\varphi, z) \doteq \sum_{\mu=M_{r}+1}^{M} \alpha_{i, \mu} \phi_{\mu}(\varphi, z)$.

Here, the first $N_{r}-L$ functions $\phi_{\mu}$ in $j_{\perp}$ are the basis functions needed to distinguish between the rings in one group; the remaining ones correspond to the trigonometric and Legendre functions in (4.2).

Substituting (4.15) in (4.12), computing the inner products with all separate basis functions and using the selfadjointness of the operator $(\mathrm{I}-\Pi)$, we obtain

$\sum_{\mu=1}^{M_{r}} \alpha_{r, \mu}\left(\phi_{\mu}, \phi_{\nu}\right)-\mathrm{i} \kappa \sum_{\mu=1}^{M_{r}} \alpha_{r, \mu}\left(\mathrm{K}_{r r} \phi_{\mu}, \phi_{\nu}\right)-\mathrm{i} \kappa \sum_{\mu=M_{r}+1}^{M} \alpha_{i, \mu}\left(\mathrm{K}_{r i} \phi_{\mu}, \phi_{\nu}\right)=\mathrm{i} \kappa \sum_{l=1}^{L} \frac{R \hat{I}_{l}}{D_{l}}\left(\mathrm{~K}_{r r} \psi_{l}, \phi_{\nu}\right)$,

for $v=1, \ldots, M_{r}$, and

$\sum_{\mu=M_{r}+1}^{M} \alpha_{i, \mu}\left(\phi_{\mu}, \phi_{v}\right)-\mathrm{i} \kappa \sum_{\mu=M_{r}+1}^{M} \alpha_{i, \mu}\left(\mathrm{K}_{i i} \phi_{\mu}, \phi_{\nu}\right)-\mathrm{i} \kappa \sum_{\mu=1}^{M_{r}} \alpha_{r, \mu}\left(\mathrm{K}_{i r} \phi_{\mu}, \phi_{\nu}\right)=0$,

for $v=M_{r}+1, \ldots, M$.

From (4.16)-(4.17), we obtain a linear set of $M$ equations from which the $M$ unknown coefficients $\alpha_{r, \mu}, \alpha_{i, \mu}$ can be determined. 


\subsection{Calculation of the matrix elements}

For the determination of the unknown coefficients, $\alpha_{r, \mu}, \alpha_{i, \mu}$ from (4.16)-(4.17), we need to calculate the matrix elements occurring in these equations. We note that two basis functions on different rings/islands are always orthogonal, because they are defined for distinct $z$-values. Moreover, two different basis functions on the same ring/island are orthogonal, because of the orthogonality of Legendre polynomials and of trigonometric functions.

The basis functions have not been normalized, and so the Gram matrix is not equal to the identity matrix. The Gram matrix is composed by the elements $\left(\phi_{\mu}, \phi_{\nu}\right)$ and, due to the orthogonality of the basis functions, is a diagonal matrix. For instance, for basis functions on rings, see (4.15), we obtain for $\mu, v \in\left\{N_{r}-L+1, \ldots, M_{r}\right\}$ and corresponding $m \geq 0, n \geq 1$, for the cosine function in (4.2),

$$
\begin{aligned}
G_{\mu \nu} & =\left(\phi_{\mu}, \phi_{\nu}\right)=\delta_{\mu \nu} \int_{z_{0}^{(q)}}^{z_{1}^{(q)}} \int_{-\pi}^{\pi} \cos ^{2}(m \varphi) P_{n}^{2}\left(\frac{z-c_{z}^{(q)}}{d_{z}^{(q)}}\right) \mathrm{d} \varphi \mathrm{d} z \\
& =\frac{2 \pi d_{z}^{(q)}}{2 n+1}\left(\delta_{m 0}+1\right) \delta_{\mu \nu} .
\end{aligned}
$$

In case of sine functions, with $m \geq 1, n \geq 1$, we obtain the same result. The first $N_{r}-L$ elements on the diagonal of $\mathbf{G}$ are obtained from the linear combination of characteristic functions; their integral calculations are trivial.

As rings and islands are disjoint, the inner products of their basis functions yield zeros in the Gram matrix. The elements of the Gram matrix corresponding to basis functions of islands, see (4.15) and (4.6), are for $\mu, v \in$ $\left\{M_{r}+1, \ldots, M\right\}$ and corresponding $m, n$,

$$
\begin{aligned}
G_{\mu \nu} & =\left(\phi_{\mu}, \phi_{\nu}\right)=\delta_{\mu \nu} \int_{z_{0}^{(q)}}^{z_{1}^{(q)}} \int_{\varphi_{0}^{(q)}}^{\varphi_{1}^{(q)}} \sin ^{2}\left(\frac{m \pi\left(\varphi-\varphi_{0}^{(q)}\right)}{2 d_{\varphi}^{(q)}}\right) P_{n}^{2}\left(\frac{z-c_{z}^{(q)}}{d_{z}^{(q)}}\right) \mathrm{d} \varphi \mathrm{d} z \\
& =\frac{2 d_{z}^{(q)} d_{\varphi}^{(q)}}{2 n+1} \delta_{\mu \nu} .
\end{aligned}
$$

For the matrix elements that include operator K, we distinguish the following three possible cases:

1. Matrix elements from basis functions of rings mutually, $\left(\mathrm{K}_{r r} \phi_{\mu}, \phi_{\nu}\right)$.

2. Matrix elements from basis functions of a ring and an island, $\left(\mathrm{K}_{r i} \phi_{\mu}, \phi_{\nu}\right)=\left(\mathrm{K}_{i r} \phi_{\mu}, \phi_{\nu}\right)$.

3. Matrix elements from basis functions of islands mutually, $\left(\mathrm{K}_{i i} \phi_{\mu}, \phi_{\nu}\right)$.

The determination of the matrix elements always amounts to the calculation of integrals of the form:

$$
A_{p, n_{1}, n_{2}}=\int_{-1}^{1} \int_{-1}^{1} f(\chi, p) P_{n_{1}}(\zeta) P_{n_{2}}(z) \mathrm{d} \zeta \mathrm{d} z
$$

where

$$
\begin{aligned}
& f(\chi, 0)=\frac{1}{2 \pi} \int_{-\pi}^{\pi} \mathcal{K}_{\varphi}(\varphi, z) \mathrm{d} \varphi=\frac{1}{4 \pi^{2}} Q_{\frac{1}{2}}(\chi), \\
& f(\chi, p)=\frac{1}{\pi} \int_{-\pi}^{\pi} \mathcal{K}_{\varphi}(\varphi, z) \cos (p \varphi) \mathrm{d} \varphi=\frac{1}{4 \pi^{2}}\left(Q_{p-\frac{3}{2}}(\chi)+Q_{p+\frac{1}{2}}(\chi)\right), \\
& \chi=\chi\left(z, \zeta ; q_{1}, q_{2}\right)=\frac{2+\left(d_{z}^{\left(q_{1}\right)} z-d_{z}^{\left(q_{2}\right)} \zeta+c_{z}^{\left(q_{1}\right)}-c_{z}^{\left(q_{2}\right)}\right)^{2}}{2} .
\end{aligned}
$$


The kernel $f(\chi, p)$, as found in (4.21)-(4.22), has a logarithmic singularity at $\chi=1$, for all $p \geq 0$, according to (3.31). For the computation of the double integral in (4.20), we first extract the logarithmic part. The remaining part is regular and can be computed numerically in a straightforward way. For the logarithmic part, an analytical expression exists; see [14, Eq. 44]. Defining

$\Pi_{k k^{\prime}}=\frac{d_{q}^{2}}{2 \pi} \int_{-1}^{1} \int_{-1}^{1} P_{k}(z) P_{k^{\prime}}(z)\left[\log \left(d_{q}|z-\zeta|\right)-\log 2+\Psi^{(0)}\left(\frac{2 p+1}{2}\right)+\gamma\right] \mathrm{d} \zeta \mathrm{d} z$,

we obtain

$$
\Pi_{k k^{\prime}}= \begin{cases}\frac{8 d_{q}^{2}}{2 \pi\left(k+k^{\prime}\right)\left(k+k^{\prime}+2\right)\left[\left(k-k^{\prime}\right)^{2}-1\right]}, & \text { if } k+k^{\prime}>0 \text { even, } \\ 0, & \text { if } k+k^{\prime} \text { odd, } \\ \frac{d_{q}^{2}}{2 \pi}\left(4 \log d_{q}-6+4 \Psi^{(0)}\left(\frac{2 p+1}{2}\right)+4 \gamma\right), & \text { if } k=k^{\prime}=0 .\end{cases}
$$

This analytical formula enables us to perform fast computations and to obtain accurate results. In the numerical examples presented in Sect. 5, Legendre polynomials up to eighth order have been used to obtain an accuracy of one percent. Now, having solved the integral from (4.20), all that remains to determine the matrix elements is elementary calculus.

\section{Numerical results}

In this section, we present numerical results for the current-density distributions in three different configurations: one ring and one island, two rings and one island, and two rings and four islands. The geometries used in the numerical examples are chosen such that they can be compared with the ring examples presented in [14]. The examples are not meant to mimic realistic $z$-gradient coils. All the computations are performed for a set of frequencies that are within the range of significant Fourier components for MRI applications. All simulations make use of the method described in this article; first, the coefficients $\alpha_{r, \mu}, \alpha_{i, \mu}$ are determined from (4.16)-(4.17), then $j_{\perp}, j_{i, \varphi}$ from (4.15), and finally $j_{r, \varphi}$ on the rings from (4.10) and the $z$-components $j_{r, z}$ and $j_{i, z}$ from (4.3) and (4.7), respectively. We also computed the total resistance and self-inductance for the three configurations.

\subsection{One ring and one island}

The first configuration that we consider consists of one ring together with one island, both on a cylinder of radius $R=0.35 \mathrm{~m}$. The width of the ring is $0.04 \mathrm{~m}$, and its center is positioned at $z=-0.04 \mathrm{~m}$. It carries a total current of $600 \mathrm{~A}$ at four different frequencies $f$, i.e., $f=100,400,700$, or $1,000 \mathrm{~Hz}$. The island has a width of $0.02 \mathrm{~m}$, and its length $l\left(l:=\left(\varphi_{1}^{(1)}-\varphi_{0}^{(1)}\right) R\right)$ is a quarter of the circumference of the cylinder, i.e., $l=2 \pi R / 4 \approx 0.55 \mathrm{~m}$. The center of the island is positioned at $(\varphi, z)=(0,0)$. Due to the time-dependent source current in the ring, eddy currents are induced in both ring and island.

In Fig. 2a, the amplitude of the current density in the $\varphi$-direction, $\left|j_{\varphi}\right|$, at $\varphi=0$ is plotted as a function of $z$, and for the four values of frequency $f$. We observe a distribution over the width of the ring that is not symmetric, in contrast to the symmetric distribution for one ring only, as can be found in [14, Fig. 3(a)]. The reason for this is that an eddy current has been induced in the island, which affects the distribution in the ring. The direction of the eddy current in the island is such that it opposes the magnetic field caused by the ring. Thus, at $z=-0.01$, this direction is opposite to the one in the ring. Opposite currents amplify each other, resulting in an amplitude of the current in the ring that is higher at $z=-0.02$ than at $z=-0.06$. 

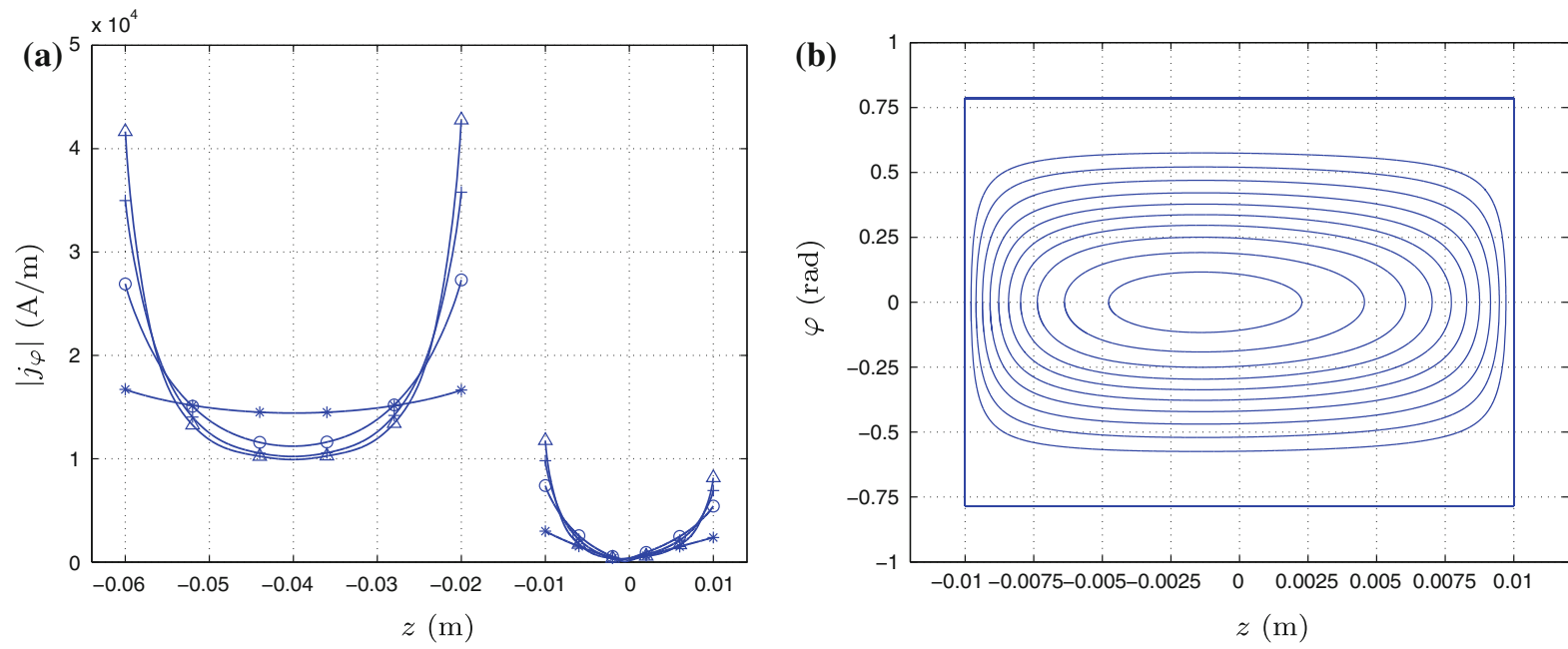

Fig. 2 One ring of $4 \mathrm{~cm}$ width with a total current of $600 \mathrm{~A}$ and one island of $2 \mathrm{~cm}$ width and $55 \mathrm{~cm}$ length. a Amplitude of the current density in the $\varphi$-direction on the line $\varphi=0$, at frequencies $f=100 \mathrm{~Hz}(*), f=400 \mathrm{~Hz}(\circ), f=700 \mathrm{~Hz}(+)$, and $f=1,000 \mathrm{~Hz}(\triangle)$; b streamlines of the current in the island at frequency $f=1,000 \mathrm{~Hz}$, with steps in $\left|j_{\varphi}\right|$ of $1,000 \mathrm{~A} / \mathrm{m}$

The current on the island being divergence free implies that the total normal current across $\varphi=0$ must be zero. Thus, $j_{\varphi}(0, z)$ integrated from $z=-0.01$ to $z=0.01$ must be equal to zero. In Fig. 2a, the amplitude $\left|j_{\varphi}(0, z)\right|$ is shown, which is greater than zero everywhere, but the sign of $j_{\varphi}(0, z)$ changes at the point where $\left|j_{\varphi}(0, z)\right|=0$, yielding indeed a zero total normal current. We observe an edge-effect in the island and an amplitude at $z=-0.01$, which is greater than at $z=0.01$, because the latter is amplified by the opposite current in the ring.

In Fig. 2b, the streamlines of the current in the island are shown. The streamlines form closed cycles, called eddies, revealing that the currents are divergence free. The distances between the streamlines represent equidistant steps of $1,000 \mathrm{~A} / \mathrm{m}$ in $\left|j_{\varphi}\right|$. In this way, the magnitude of the edge-effect in Fig. $2 \mathrm{~b}$ is represented by the density of the streamlines. Note that the eye (i.e., the center) of the eddy is not at the center of the island, but closer to the ring, in agreement with the fact that on that side the induced current is stronger.

The current in the ring is mainly in the $\varphi$-direction. In contrast to the configuration of one ring only, the current also has a $z$-component, induced by the island. However, compared to $j_{\varphi}, j_{z}$ is very small. Therefore, the streamlines in the ring would be straight lines on the scale of this figure; cf. Fig. 5b, further on.

For low frequencies (quasi-static range), the behavior of $j_{\varphi}$ near the edges is regular, as described in [13]. In case of high frequencies, a square-root singular edge-effect will occur in the current near the edges. This is common in the RF coils used for MRI, but not in gradient coils.

\subsection{Two rings and one island}

In the second configuration that we consider, we put an additional ring to the right of the island. This ring also has a width of $0.04 \mathrm{~m}$, and is positioned with its center at $z=0.04 \mathrm{~m}$. This configuration is chosen such that it can be compared with the two-rings case presented in [14]. Both rings carry a current of $600 \mathrm{~A}$.

First, we consider the case that the two source currents in the rings are in anti-phase. In Fig. 3a, the amplitude of the current density in the $\varphi$-direction on $\varphi=0$ is shown as a function of $z$ and for four different frequencies. An eddy current is induced in the island, which also induces eddy currents in the rings. Comparing Fig. 3a with Fig. 2a, we see that the induced current in the island is symmetric and almost twice as strong, in conformity with our expectations. Moreover, the asymmetry in the edge-effects in the rings is much more pronounced here.

In Fig. 3b, streamlines of the current in the island are shown, where $\left|j_{\varphi}\right|$ makes steps of $2,000 \mathrm{~A} / \mathrm{m}$. We observe one eddy with its eye at the center of the island; the current distribution is symmetric in both $\varphi$ and $z$. 

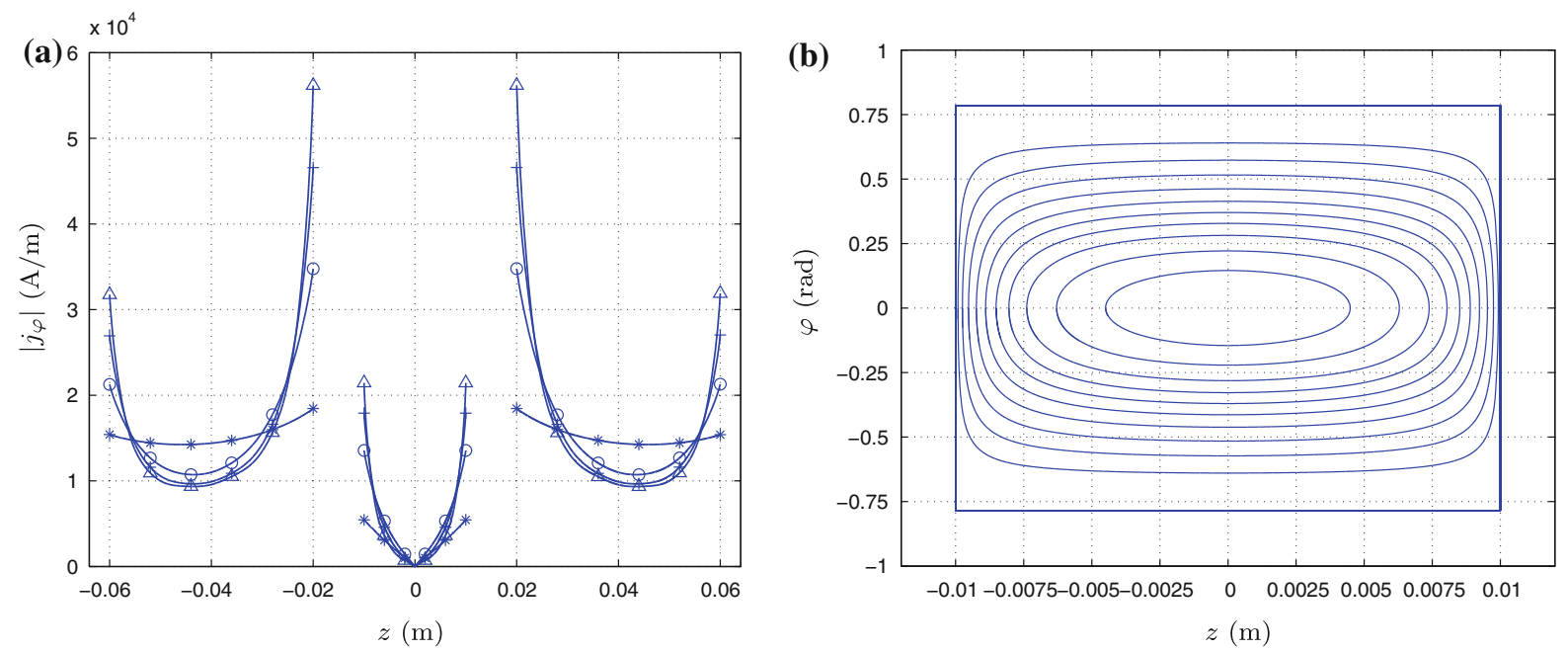

Fig. 3 Two rings of $4 \mathrm{~cm}$ width with total currents of $600 \mathrm{~A}$ in anti-phase and one island of $2 \mathrm{~cm}$ width and $55 \mathrm{~cm}$ length in between them. a Amplitude of the current density in the $\varphi$-direction on the line $\varphi=0$, at frequencies $f=100 \mathrm{~Hz}(*), f=400 \mathrm{~Hz}(\circ), f=700 \mathrm{~Hz}$ $(+)$, and $f=1,000 \mathrm{~Hz}(\triangle)$; b streamlines of the current in the island at frequency $f=1,000 \mathrm{~Hz}$, with steps in $\left|j_{\varphi}\right|$ of $2,000 \mathrm{~A} / \mathrm{m}$
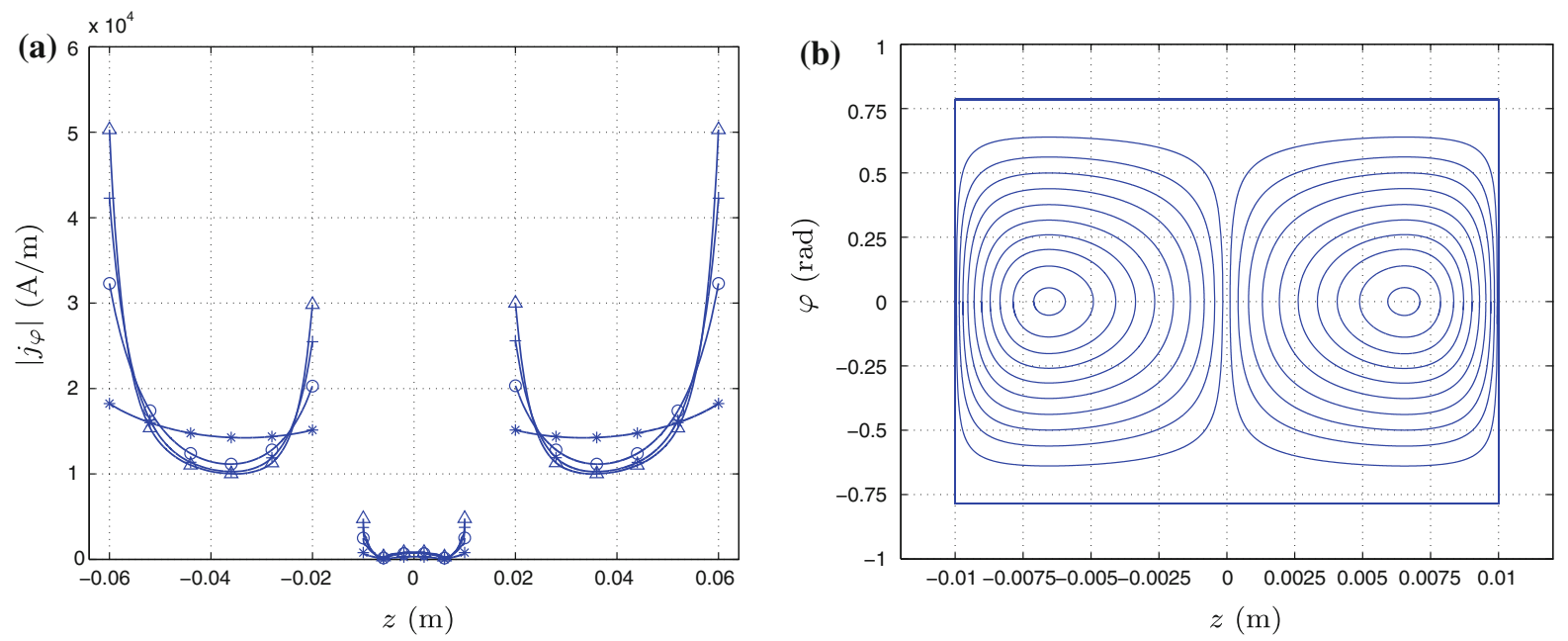

Fig. 4 Two rings of $4 \mathrm{~cm}$ width with total currents of $600 \mathrm{~A}$ in phase and one island of $2 \mathrm{~cm}$ width and $55 \mathrm{~cm}$ length in between them. a Amplitude of the current density in the $\varphi$-direction on the line $\varphi=0$, at frequencies $f=100 \mathrm{~Hz}(*), f=400 \mathrm{~Hz}(\circ), f=700 \mathrm{~Hz}(+)$, and $f=1,000 \mathrm{~Hz}(\triangle)$; b streamlines of the current in the island at frequency $f=1,000 \mathrm{~Hz}$, with steps in $\left|j_{\varphi}\right|$ of $300 \mathrm{~A} / \mathrm{m}$

Second, in Fig. 4, the results are shown for the situation in which the currents in the rings are in phase. From Fig. 4a, we observe local edge-effects in the rings and in the island, and a global edge-effect in the whole system. A striking difference with the previous case is that the larger edge-effects are now at the far (far away from the island) edges of the rings. The observed edge-effects are a consequence of the physical phenomenon that currents in the same direction repel. Currents in opposite direction attract as can be observed in the rings of Fig. $3 \mathrm{a}$.

Moreover, the current in the island in this case is smaller than in the previous example. The reason for this is that now, in order to maintain the symmetry in the system, two eddies are induced on the island. These two eddies are visualized by the streamlines in Fig. $4 \mathrm{~b}$, where $\left|j_{\varphi}\right|$ makes steps of $300 \mathrm{~A} / \mathrm{m}$. The edge-effects are also visible in this figure: we observe the eddies with eyes close to the $z$-edges of the island. 

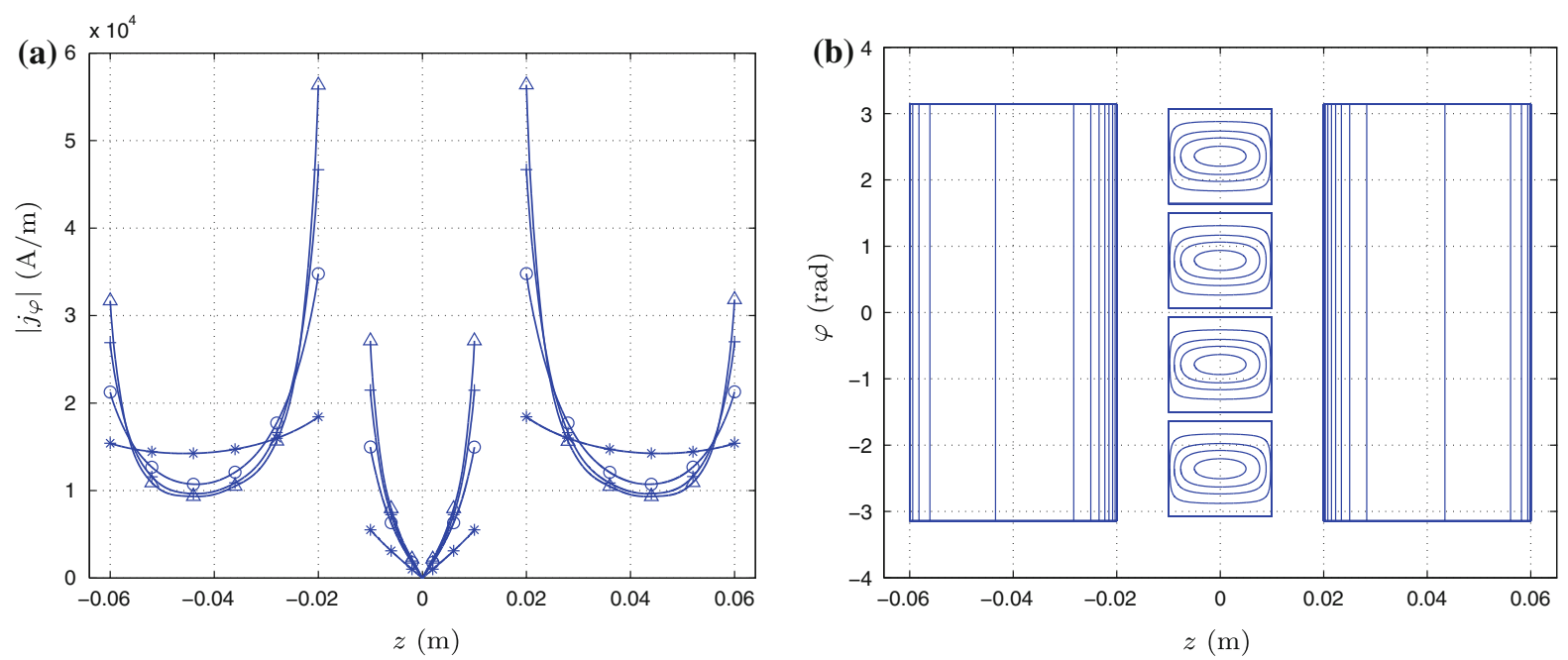

Fig. 5 Two rings of $4 \mathrm{~cm}$ width with total currents of $600 \mathrm{~A}$ in anti-phase and four islands of $2 \mathrm{~cm}$ width and $50 \mathrm{~cm}$ length between them. a Amplitude of the current density in the $\varphi$-direction on the line $\varphi=\pi / 4$, at frequencies $f=100 \mathrm{~Hz}(*), f=400 \mathrm{~Hz}(\circ), f=700 \mathrm{~Hz}$ $(+)$, and $f=1,000 \mathrm{~Hz}(\triangle)$; $\mathbf{b}$ streamlines of the currents at frequency $f=1,000 \mathrm{~Hz}$, with steps in $\left|j_{\varphi}\right|$ of $6.0 \cdot 10^{3} \mathrm{~A} / \mathrm{m}$

\subsection{Two rings and four islands}

In the third configuration, we consider two rings at the same positions as in the previous example, but now with four islands in between. The four islands have their centers at $z=0$ and each island has a length of $0.5 \mathrm{~m}$. This means that all together they almost form a complete ring, but cut in four pieces. Note that this is an abstract example, which does not occur in a realistic $z$-gradient coil. The source currents are applied to the rings, are in anti-phase, and have an intensity of $600 \mathrm{~A}$.

In Fig. 5a, the amplitude of the current density in the $\varphi$-direction on the line $\varphi=\pi / 4$ is shown. This line crosses the center of one of the islands. Due to symmetry, the current distributions are the same in all four islands. The current distribution in the rings is similar to that in the previous example with one island; see Fig. 3a. Compared with the distribution in one island, the currents in each of the four islands have a higher amplitude. The reason for this is that the currents in the islands also amplify each other.

In Fig. 5b, streamlines of the currents in the rings and in the islands are shown. The streamlines in the rings are straight, indicating that the induced $z$-component of the current is much smaller than the $\varphi$-component. We observe that in each island one eddy appears. Here, $\left|j_{\varphi}\right|$ makes steps of $6.0 \times 10^{3} \mathrm{~A} / \mathrm{m}$, both for the rings and the islands. The eyes of the eddies are at the centers of the islands due to symmetry.

An interesting aspect in this example is that the four islands behave qualitatively as one ring. Namely, on the edges between the two neighboring islands, the currents are in opposite direction, such that the resulting magnetic fields from these currents cancel each other. Moreover, on $z=-0.01$ and $z=0.01$, the currents are in the same direction. Therefore, from a certain distance, the currents are observed as distributed in a ring. Consequently, the effect of the slits on the magnetic field at some distance from the surface of the cylinder is negligible.

\subsection{Resistance and self-inductance}

Apart from the magnetic field, the islands also affect the resistance and the self-inductance of the gradient coil. For the computation of the resistance and the self-inductance, we introduce the $M \times M$-matrices $\mathbf{G}$ and $\mathbf{A}$ and the $M$-dimensional column vector a. Here, $\mathbf{G}$ denotes the diagonal Gram matrix with the inner products of the separate basis functions $\left(\phi_{\mu}, \phi_{\nu}\right)$ as diagonal elements. The elements of matrix $\mathbf{A}$ are represented by the inner 
products $\left(\mathrm{K}_{r r} \phi_{\mu}, \phi_{v}\right),\left(\mathrm{K}_{r i} \phi_{\mu}, \phi_{v}\right),\left(\mathrm{K}_{i r} \phi_{\mu}, \phi_{\nu}\right),\left(\mathrm{K}_{i i} \phi_{\mu}, \phi_{v}\right)$. The vector a consists of the $M$ coefficients of the basis functions. The time-averaged dissipated power (see [16, Sect. 2.19]) can be expressed in terms of $\mathbf{G}$ and a as

$\bar{P}_{\text {diss }}=\frac{R^{2}\left|j^{c}\right|^{2}}{2 h \sigma} \iint_{S \cup} \mathbf{j}(\mathbf{x}) \cdot \mathbf{j}^{*}(\mathbf{x}) \mathrm{d} \theta(\mathbf{x}) \mathrm{d} \zeta(\mathbf{x})=\frac{R^{2}\left|j^{c}\right|^{2}}{2 h \sigma} \mathbf{a}^{*} \mathbf{G}$,

where $\mathbf{a}^{*}$ is the complex conjugate of $\mathbf{a}$.

Moreover, the effective current $I_{e}$ is defined as

$I_{e}^{2}=I I^{*} / 2=2\left|j^{c}\right|^{2} R^{2} d_{z}^{2}$,

with $d_{z}$ half the (dimensionless) width of a ring. Thus, for the resistance $\bar{R}$ we obtain:

$\bar{R}=\frac{\bar{P}_{\mathrm{diss}}}{I_{e}^{2}}=\frac{1}{4 h \sigma d_{z}^{2}} \mathbf{a}^{*} \mathbf{G a}$.

The time-averaged magnetic energy (see, e.g., [16, Sect. 2.19]) can be expressed in terms of $\mathbf{A}$ and $\mathbf{a}$ as

$\bar{U}_{m}=\frac{\mu_{0} R^{3}\left|j^{c}\right|^{2}}{16 \pi} \iint_{S} \iint_{S} \frac{\mathbf{j}(\boldsymbol{\xi}) \cdot \mathbf{j}^{*}(\mathbf{x})}{|\mathbf{x}-\boldsymbol{\xi}|} \mathrm{d} \theta^{\prime}(\boldsymbol{\xi}) \mathrm{d} \zeta^{\prime}(\boldsymbol{\xi}) \mathrm{d} \theta(\boldsymbol{x}) \mathrm{d} \zeta(\boldsymbol{x})=\frac{\mu_{0} R^{3}\left|j^{c}\right|^{2}}{4} \mathbf{a}^{*} \mathbf{A} \mathbf{a}$.

The self-inductance $\bar{L}$ is then computed from

$\bar{L}=\frac{\bar{U}_{m}}{\frac{1}{2} I_{e}^{2}}=\frac{\mu_{0} R}{4 d_{z}^{2}} \mathbf{a}^{*} \mathbf{A a}$.

In Fig. 6, we show the resistance and the self-inductance as a function of the frequency, for the following four different configurations.

First: two rings with a width of $0.04 \mathrm{~m}$ and a distance between the centers of $0.08 \mathrm{~m}$. The currents in the rings are in anti-phase. The resistance $\bar{R}_{D C}$ at $f=0$ can be computed analytically from

$\bar{R}_{D C}=\frac{4 \pi R}{h D \sigma} \approx 7.48 \times 10^{-4} \Omega$.

The resistance increases with the frequency; see the lowest curve in Fig. 6a, where the frequency range is up to $f=1,0000 \mathrm{~Hz}$.

Second: an island placed between the two rings. This configuration is the same as in the first (anti-phase) example of Sect. 5.2. In case of a stationary current, no eddy currents are induced. In other words, the system does not notice the presence of the island then. Therefore, the resistance $\bar{R}_{D C}$ at $f=0$ is the same as for two rings only. Due to the eddy currents appearing in the island for $f>0$, the resistance increases a bit more with the frequency than in the first case; see the second lowest curve in Fig. $6 \mathrm{a}$.

Third: the configuration of two rings and four islands. The resistance $\bar{R}_{D C}$ at $f=0$ is the same as in the previous two configurations. For higher frequencies, eddy currents are induced in all four islands. Together, they oppose the magnetic field more than the eddies in one island, causing the total resistance of the system to be higher. This is shown by the second highest curve in Fig. 6 a.

Fourth: the configuration of the two rings from the first case, with an additional ring in between them, not connected to any source. Again, for a stationary current in the two rings that are connected to the source, no eddy currents are induced, and so again $\bar{R}_{D C}=7.48 \times 10^{-4} \Omega$. For higher frequencies, a current $j_{\varphi}(z),\left(j_{z}=0\right)$ is induced in the additional ring having an anti-symmetrical current distribution in $z$. At the right (left) edge of this ring, the current is opposite to that at the right (left) neighboring ring. As a consequence of this anti-symmetric current distribution, the total current in the additional ring is equal to zero. In contrast to the third case with the separated islands, here the current on the additional ring forms closed cycles. However, due to these closed cycles 

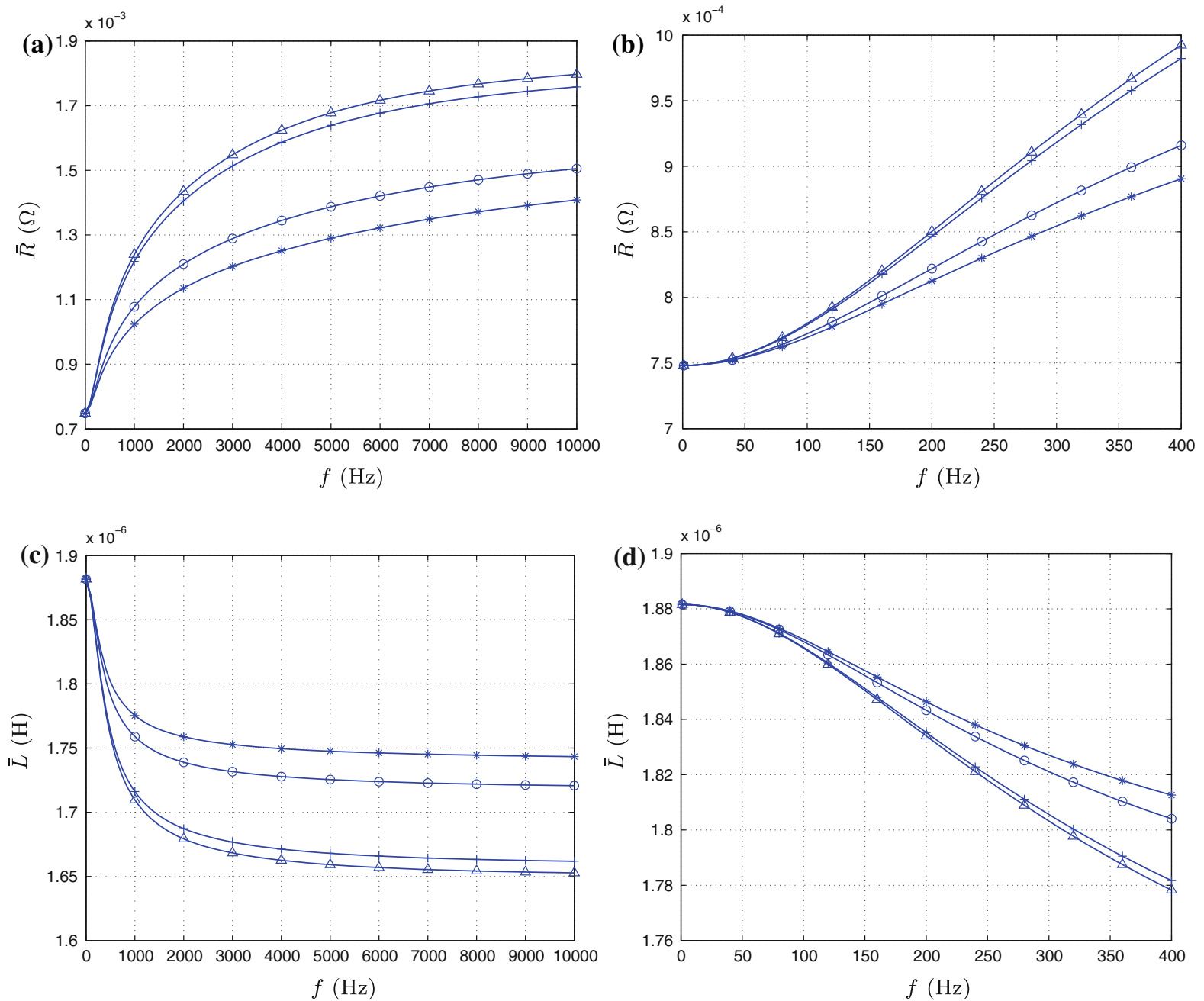

Fig. 6 Resistance and self-inductance of a set of two rings (*), a set of two rings with one island in between (o), a set of two rings with four islands in between $(+)$, and a set of three rings $(\triangle)$. The source currents in the two outer rings are always in anti-phase. a $\bar{R}$ at frequencies up to $f=10,000 \mathrm{~Hz} ; \mathbf{b} \bar{R}$ at frequencies up to $f=400 \mathrm{~Hz} ; \mathbf{c} \bar{L}$ at frequencies up to $f=10,000 \mathrm{~Hz}$; and $\mathbf{d} \bar{L}$ at frequencies up to $f=400 \mathrm{~Hz}$

of the current, the magnetic field is more opposed than in the previous configurations and the total resistance of the system is higher; see the highest curve in Fig. 6a.

In Fig. 6b, the resistance is shown for the smaller range of frequencies up to $f=400 \mathrm{~Hz}$, with a higher resolution than used in the range from 0 to $10,000 \mathrm{~Hz}$. All points of inflection in the resistances of the four configurations lie in this range. In Fig. 6c, the self-inductances of the four configurations are shown for the frequency range up to $f=10,000 \mathrm{~Hz}$. We observe that the self-inductances decrease with the frequency and that the order of the curves is opposite to the order in the figure of the resistances, i.e., the two-ring case is represented by the highest curve, and the three-ring case is represented by the lowest curve. The self-inductances in the smaller range from 0 to 400 $\mathrm{Hz}$ are shown in Fig. 6d. All points of inflection in the self-inductance of the four configurations lie in this range. As shown in [14, Sect.4.1], the frequency associated with this inflection point, say $f_{\text {char }}$, represents the frequency at which prevailing resistive effects with respect to inductive effects ( $f<f_{\text {char }}$ ) change into prevailing inductive effects $\left(f>f_{\text {char }}\right)$. 


\section{Conclusions}

This article describes a model for the current distribution in a set of rings and islands on a cylindrical surface. A Fredholm integral equation of the second kind has been derived for the current density distribution in the strips. The kernel function has a logarithmic singularity in the axial coordinate and can be expressed in terms of Legendre functions of the second kind of odd-half-integer order. The asymptotic expansion of these functions near the singular point reveals the logarithmic function in the kernel. The Fredholm integral equation was solved by the Galerkin method: the basis functions used are Legendre polynomials and $2 \pi$-periodic trigonometric functions. To perform the integrations needed here, the logarithmic part of the kernel is split off and integrated analytically, while the remaining regular part was solved by straightforward numerical integration. This makes our implementation very efficient from a computational point of view.

For the logarithmic part, we used the analytical formula given by (4.25), where Legendre polynomials are used as basis functions in the axial direction. This analytical result yields a very fast algorithm, for which only very few Legendre polynomials are needed, because convergence is fast. In the tangential direction, Fourier modes were used, because of the $2 \pi$-periodicity and the orthogonality with the trigonometric functions in the expansion of the kernel function. In the resulting set of equations, the mutual interaction between the rings and the islands was explicitly expressed by the coupling between the modes.

Numerical simulations have been performed for three configurations: two rings, two rings with one island, and two rings with four islands. The source currents were applied to the two outer rings. These are always in anti-phase, except for the second configuration, where in-phase source currents also are considered. The resulting current density distribution shows the occurrence of eddy currents and edge-effects in both rings and islands. In the antiphase cases, the edge-effects are always most pronounced at the inner edges of the rings, whereas in the in-phase case, this is observed at the outer edges. The magnitude of the edge-effects increases with the number of islands, because of the larger eddy currents. However, in the in-phase case, the eddy current in the island is very small, and consequently, the edge-effects in this case are smaller than those in the corresponding anti-phase case. In the third configuration, a full ring is cut into four islands by four slits. From the resulting current-density distributions, we infer that the differences between four islands and a full ring are small. Consequently, the internal magnetic field of the gradient coil near the axis of the MRI-scanner is hardly affected by the slits.

We have shown that the total resistance of a system of strips on a cylinder increases when an island is added to the system, whereas the opposite holds for the self-inductance of the system. When a ring, not connected to a source, is added to the system, the resistance increases even more, whereas the self-inductance decreases even more. This additional ring is a good approximation of a full set of islands, as such describing a ring with slits.

The resulting simulation tool has proven to be an appropriate extension of the tool that models current distributions in rings [14]. It can be used to qualitatively understand eddy currents in gradient-coil designs, as it can indicate trends in the sensitivity to the positions of the strips and the model parameters used with respect to the desired gradient fields.

Logical future extensions of our study are in the computation of the created magnetic gradient field (for this, classical methods involving spherical harmonics can be used) and the application to magnetic shielding. For the latter, our approach could be applied to a set of two concentric cylinders covered with conducting strips. The mutual interaction between the two cylinders must then be taken into account.

Open Access This article is distributed under the terms of the Creative Commons Attribution Noncommercial License which permits any noncommercial use, distribution, and reproduction in any medium, provided the original author(s) and source are credited.

\section{References}

1. Vlaardingerbroek MT, den Boer JA (1999) Magnetic resonance imaging. Springer, Berlin

2. Jin JM (1999) Electromagnetic analysis and design in magnetic resonance imaging. CRC Press, London

3. Tegopoulos JA, Kriezis M (1985) Eddy currents in linear conducting media. Elsevier, Amsterdam 
4. Stoll RL (1974) The analysis of eddy currents. Clarendon Press, Oxford

5. Lammeraner J, Stafl M (1966) Eddy currents. Hiffe books, London

6. Roméo F, Hoult DI (1984) Magnetic field profiling: Analysis and correcting coil design. Magn Reson Med 1:44-65

7. Suits H, Wilken DE (1989) Improving magnetic field gradient coils for NMR imaging. J Phys E 22:565-573

8. Turner R (1986) A target field approach for optimal coil design. J Phys D 19:147-151

9. Wong E, Jesmanowicz A (1991) Coil optimization for MRI by conjugate gradient descent. Magn Reson Med 21:39-48

10. Crozier S, Doddrell DM (1993) Gradient coil design by simulated annealing. J Magn Reson 103:354-357

11. Tomasi D (2001) Stream function optimization for gradient coil design. Magn Reson Med 45:505-512

12. Trakic A, Liu F, Sanchez Lopez H, Wang H, Crozier S (2007) Longitudinal gradient coil optimization in the presence of transient eddy currents. Magn Reson Med 57(6):1119-1130

13. Ulicevic T, Kroot JMB, van Eijndhoven SJL, van de Ven AAF (2005) Current distribution in a parallel set of conducting strips. J Eng Math 51:381-400

14. Kroot JMB, van Eijndhoven SJL, van de Ven AAF (2007) Eddy currents in a gradient coil, modeled as circular loops of strips. J Eng Math 57:333-350

15. Kroot JMB, van Eijndhoven SJL, van de Ven AAF (2008) Eddy currents in a transverse MRI gradient coil. J Eng Math 62:315-331

16. Stratton JA (1941) Electromagnetic theory. McGraw-Hill, London

17. Sneddon IN (1951) Fourier transforms. McGraw-Hill, New York

18. Luke YL (1962) Integrals of Bessel functions. McGraw-Hill, New York

19. Watson GN (1922) A treatise on the theory of Bessel functions: tables of Bessel functions. Cambridge University Press, Cambridge

20. Magnus W, Oberhettinger F, Soni RP (1966) Formulas and theorems for the special functions of mathematical physics. Springer, Berlin

21. Spanier J, Oldham KB (1987) An atlas of functions. Springer, Berlin

22. Abramowitz M, Stegun IA (1968) Handbook of mathematical functions with formulas, graphs and mathematical tables. Dover Publications, New York

23. Golberg MA, Chen CS (1997) Discrete projection methods for integral equations. Computational Mechanics Publications, Southampton

24. Kreyszig E (1978) Introductory functional analysis with applications. Wiley, New York 